\title{
A simulation-based optimization approach for the calibration of dynamic train speed profiles
}

\author{
Nikola Bešinović, Egidio Quaglietta, Rob M.P. Goverde \\ Department of Transport and Planning, Delft University of Technology \\ P.O. Box 5048, 2600 GA Delft, The Netherlands \\ e-mail: \{n.besinovic,e.quaglietta,r.m.p.goverde\}@ tudelft.nl
}

\begin{abstract}
Predictions of railway traffic are needed by planners and dispatchers for the design of robust timetables and real-time traffic management of perturbed conditions. These tasks can be effectively performed only when using train running time models which reliably describe actual speed profiles. To this purpose calibration of model parameters against field data is a necessity. In this paper a simulation-based optimization approach is introduced to calibrate the parameters of the train dynamics equations against field data collected at the level of track sections. A genetic algorithm is used to minimize the error between simulated and observed speed profiles. Furthermore, a procedure for the estimation of train lengths has been developed. This method has been applied to trains with different rolling stock running on the Rotterdam-Delft corridor in the Netherlands. The model parameters were calibrated for a significant number of trains of different compositions. We also derived probability distributions for each parameter which can be usefully employed for simulations. The results show that the train length estimation model obtained good computation accuracy. The effectiveness of the calibration method in giving a reliable estimation of the real train path trajectories is shown. It has been observed that some of the parameters of tractive effort and resistance do not affect the train behaviour significantly. Also, the braking rate is significantly smoother than the default value used by the railway undertaking while calibrated resistance parameters tend to have lower mean than defaults. Finally, the computational efficiency of the approach is suitable for real-time applications.
\end{abstract}

\section{Keywords}

Train dynamics, running time models, calibration, simulation-based optimization, speed profile estimation

\section{Introduction}

Recent demand growth for passenger and freight transportation in railway systems has raised the need for practitioners to increase the level of network capacity while keeping a high standard of service availability and quality. To achieve this aim railway traffic needs to be scheduled according to robust timetables that guarantee higher levels of capacity usage also in presence of stochastic disturbances. On the other hand, suitable control measures (e.g. train retiming, reordering and/or rerouting) must be taken in real-time by dispatchers to provide rescheduling plans that mitigate the effects of observed conflicts on network performances. Both robust timetabling and real-time management of railway traffic aim at supplying conflict-free train path plans computed on the basis of off-line and on-line predictions of traffic behaviour. In the first step, train trajectories must be computed taking into account microscopic details of the infrastructure (e.g. lengths, 
gradient, curvature of rail tracks, speed limits), signalling system (e.g. positions of signals, block section lengths, braking behaviour imposed by the automatic train protection), train composition (e.g. number of wagons, rolling stock characteristics), and current traffic information when the prediction is performed on-line. Then, based on the estimated train trajectory a conflict-free schedule is constructed by solving a mathematical problem (e.g. optimization, heuristics), or by relying on rule-of-thumbs or the own experience of the operator (i.e. a planner in timetabling and a dispatcher within real-time operations). The effectiveness of these schedules depends on the reliability of the estimated train trajectories and the precise identification of potential track conflicts. Inaccurate forecasts can lead to wrong detection of possible conflicts and to traffic schedules that are ineffective or even infeasible when put into operation. In this context, accurate traffic prediction models must be used to confidently describe the real evolution of train behaviour. To this purpose a proper calibration phase is needed to fine-tune input parameters against train data (e.g. position, speed) collected from the field, so that the model can reproduce the real train trajectories as much as possible.

This paper presents an approach to derive the most probable speed profiles of train runs from observed track occupation/release data. The train behaviour is modelled according to the Newton dynamic motion equations which are numerically integrated over distance employing the Runge-Kutta method [4]. A simulation-based optimization approach is adopted to calibrate input parameters of the equations describing the tractive effort, the motion resistances, the braking effort, and the cruising phase. These parameters are fine-tuned for different classes of train composition (defined by the number of wagons, the type of traction unit, and the length of the train) by minimizing the gap between observed and simulated running times, using a genetic algorithm. Additionally, due to unknown type of composition beforehand, the model for train length estimation is developed. For each composition the calibration experiment is performed over a significant set of observed train runs. This enabled estimating the probability distributions the different input parameters for each class of train compositions. This aspect gives also insight in different driving behaviour adopted during real operations. The proposed approach is applied to train runs operating along the corridor Rotterdam-Delft in the Netherlands. Results illustrate the effectiveness of this method in calibrating parameters of the Newton's dynamic equations versus track occupation/release data collected at the level of track sections.

Section 2 gives a literature review on the different approaches proposed to model train running times and calibrate model parameters. In section 3 , the methodology proposed in this paper is described. Section 4 illustrates the case study considered for the application and provides the corresponding results. Conclusions and final comments are given in section 5 .

\section{Literature review}

In the literature, several approaches are presented for estimating train running times taking into account microscopic features of both trains and the infrastructure (including the signalling system). In particular, models can be mainly divided in the ones using kinematic motion equations and others adopting a dynamic representation of the movement, basically by means of Newton's motion formula [7].

Albrecht et al. [2], [3] described train motion based on the kinematic equations and calibrate their parameters (speed and acceleration) versus track occupation data collected by means of train describer systems [5], [6]. Albrecht et al. [1] use calibrated kinematic 
models to understand the influence of the Dutch signalling and ATP system on train speed profile and energy consumption. The disadvantage of these models is that they calibrate only the parameters of the kinematic motion equations which are trajectory-dependent and cannot be used anymore when considering a different train run even if the rolling stock is the same.

Kecman and Goverde [10] adopt a method suitable for real-time predictions, that represents train trajectories by means of a weighted graph that evolves dynamically each time that new information is gathered from the field; weights of the arcs are train running times measured by means of detailed track occupation/release data from train describer records collected at the level of track sections (e.g. axle counters, track circuits).

Medeossi et al. [12] use a dynamic equation for each phase of the train motion (i.e. acceleration, cruising, coasting and braking) and fine-tunes the respective performance parameters against GPS data collected on-board of the trains. A probability distribution is then estimated for these parameters to characterize stochastic variations of running times.

Hertel and Steckel [8] developed a stochastic model for the estimation of train running times and proposed to replace the deterministic running time supplements by a high percentile of simulated running time distributions at a given level of significance.

During real operations stochastic variations to individual train runs are observed due to changes in the rolling stock condition, rail deterioration, as well as variations in the train driver behaviour and weather circumstances. These unpredictable variations induce an alteration of train characteristics such as the deceleration and the acceleration rates as well as motion resistances (e.g. due to gradient, air viscosity, rail curvatures) and consequently, a change in train trajectories [10]. According to this, approximated parameters estimated by manufacturer or train operators should not be taken for granted [15], but need to be computed for each train composition and railway corridor separately.

To the best of the authors' knowledge only scarce efforts have been addressed in literature to the estimation of parameters relative to tractive effort and motion resistances based on actual train data. The present paper gives a contribution in this direction.

\section{Methodology}

\subsection{A simulation-based framework to calibrate dynamic equations of train motion}

To provide a reliable prediction model able to accurately reproduce real train trajectories it is necessary to calibrate model parameters against real data collected from the field. In this paper the calibration process is performed by developing a simulation-based framework that integrates a genetic algorithm with a microscopic running time model based on dynamic motion equations as given by Newton.

This framework has been developed in Matlab and consists of several components (Figure 1). The entire framework is based on data relative to the infrastructure (e.g. track length, gradient, speed limits, signal and station positions), the rolling stock features (e.g. train length) and the track occupation/release collected from the field. A pre-processing phase is necessary to convert the different input data into a suitable format and combine them in order to derive information needed to initialize the calibration model. In particular these data are combined to identify the exact route (i.e. the sequence of track sections, switches, signals, and stations crossed by the train during its run) and the train length (which is related to the composition) of each observed train run. Train length has been used to group the observed train runs in different classes of train compositions. Parameters of the running time model are estimated separately for each class.

Track occupation/release data are also processed to derive for each observed run 
discrete space-time trajectory data that are used to evaluate the objective function at eachiteration of the optimization algorithm. The calibration experiment is performed only against distance-time data relative to unhindered trains, thus train runs that are not disturbed by the presence of other trains on the network. This assumption consents to understand how the value of train parameters varies over different runs due only to the behaviour of the train driver and not to the interactions with other trains.

The proposed algorithm developed for the optimization problem is customised genetic algorithm which is implemented in Matlab. Output of the framework consists of: calibrated parameters of the dynamic equation for each train (i.e. braking rate, parameters of the tractive effort equation, coefficients of the resistance equation, speed adopted in cruising phases) and the corresponding train trajectories (i.e. distance-time diagrams, speed-time and speed-distance graphs).

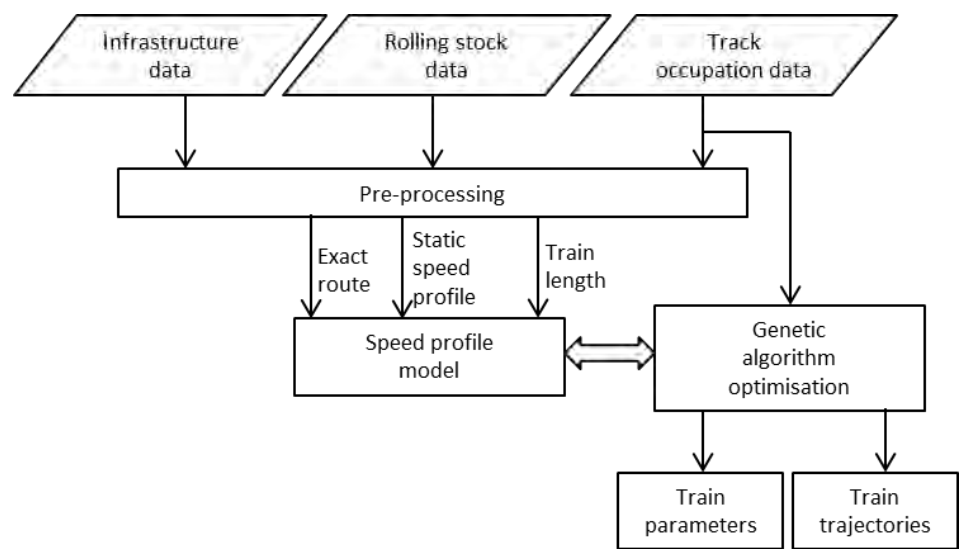

Figure 1. Functional scheme of the simulation-based optimization framework

This framework has been applied to calibrate a significant set of train runs for each class of train compositions. In this way it has been possible to estimate the probability distribution relative to the input parameters of the running time model for each train class.

\subsection{Input data}

Input data to the proposed framework are relative to the infrastructure characteristics, the rolling stock features and observed track occupation/release data collected at the level of track sections for a significant set of train runs. In this section a detailed description of each of these data is provided as follows:

- Infrastructure data contains detailed information about microscopic characteristics of railway network. These data describe lengths of track sections, curvature radii, gradients, static speed limits, positions of stations, signals and switches as well as the braking behaviour and the supervised speed codes by the Automatic Train Protection system (in this case represented by the Dutch ATB). All this information is derived from infrastructure maps and digital InfraAtlas data (version 2011) provided by the Dutch infrastructure manager (IM) ProRail.

- Rolling stock data specify all the feature regarding rail vehicles and: train compositions (number of wagons, type of traction unit), mass, parameters of the tractive effort-speed curve as well as coefficients of the resistance equations. Such 
data have been supplied by the main Dutch railway Undertaking (RU) Netherlands Railways (NS).

- Track occupation/release data are gathered from field measurements and return the time in which a given train has occupied or released a certain section on the network. This information has been collected by means of the train describer system in the Netherlands called TROTS [14]. This system logs generated train number messages and incoming infrastructure messages (from signals, switches, track sections), to provide a list of events (called train number steps obtained by coupling the train number with infrastructure messages) in a chronological order. The advantage of the TROTS system is that it is able to record train number steps at the level of track sections. Measured occupation/release times are rounded down to the full second and are affected by a systematic error of track circuits (especially in the release phase). This measurement inaccuracy has a big influence on very short sections when short occupation times are observed. These data are preprocessed by using the data mining tool developed by Kecman and Goverde, which features are explained in [9].

\subsection{Data pre-processing}

The main function of the pre-processing phase is to: i) convert the different input data into a format that is usable by the developed framework, ii) combine these data in order to derive additional information which are needed to initialize and apply the calibration model. Specifically, the latter process is addressed to provide for each observed train run: the exact route and the train length.

- The route of a train is defined as the sequence of infrastructure elements (i.e. track sections, switches, signals, station platforms) traversed during its run. To determine the route relative to a certain observed train run, it is necessary to combine track occupation/release data corresponding to that run together with the infrastructure data (InfraAtlas maps). The track occupation/release data is a chronological ordered list of the IDs (identification number) relative to the infrastructure elements crossed by the train during a certain run. By coupling this list of IDs with the infrastructure data it is possible to identify the route followed by that run in terms of length of track sections, gradients, static speed limits, curvature radii, the switches used, the signals approached, and the platforms at which it stopped.

- The train length has been estimated on the basis of track occupation/release data. To explain this procedure it is possible to refer to the example illustrated in Figure 2 where two track sections $s_{i}$ are represented together with their respective section joints $x_{i}$ and $x_{i+1}$. The average speed of train run $j$ when traversing track section $s_{i}$ can be calculated as:

$$
\bar{v}_{i, j}=\frac{x_{i+1}-x_{i}}{t^{\text {occupy }}\left(s_{i+1}\right)-t^{\text {occupy }}\left(s_{i}\right)}[\mathrm{m} / \mathrm{s}]
$$

where $t^{\text {occupy }}\left(s_{i+1}\right)$ and $t^{\text {occupy }}\left(s_{i}\right)$ represent the time in which the head of the train enters track section $s_{i}$ and $s_{i+1}$, respectively. The length $l_{j}$ of train $j$ is consequently assessed as:

$$
l_{j}=\bar{v}_{i, j} \cdot\left(t^{\text {release }}\left(s_{i}\right)-t^{\text {occupy }}\left(s_{i+1}\right)\right)[m],
$$


where $\left(t^{\text {release }}\left(s_{i}\right)\right.$ is the time instant in which the tail of the train releases track section $s_{i}$ while $t^{\text {occupy }}\left(s_{i+1}\right)$ is the instant in which the head of the train enters section $s_{i+1}$.

As said in section 3.2, due to round-off and system errors of track circuits both the occupation and release times are measured with an accuracy of $\delta$ seconds (i.e.

$t^{\text {occupy }} \pm \delta$ and $t^{\text {release }} \pm \delta$ ). This implicates that the average speed $\bar{v}_{i, j}$ and the train length $l_{j}$ are respectively assessed with an accuracy of $\Delta[\mathrm{m} / \mathrm{s}]$ and $\lambda[\mathrm{m}]$. The objective of this procedure is therefore to estimate the intervals $\left(\bar{v}_{i, j} \pm \Delta\right)$ and $\left(l_{j} \pm \lambda\right)$.

\begin{tabular}{cccc} 
& $s_{i}$ & $s_{i+1}$ & \\
\hline$x_{i}$ & $x_{i+1}$ & & $x_{i+2}$
\end{tabular}

Figure 2. Track sections and respective joints

Assume that from the analysis of rolling stock data we observe four different train compositions $c_{1}, c_{2}, c_{3}$ and $c_{4}$ with associated lengths $l_{1}, l_{2}, l_{3}, l_{4}$, respectively. The composition assigned to train run $j$ corresponds to the one whose length falls in the interval $\left(l_{j} \pm \lambda\right)$. For example, if a certain train length $l_{i}$ falls in such interval, that train run $i$ is assigned to a corresponding composition $c_{j}$. When the estimated interval $l_{j} \pm \lambda$ is wide, it can happen that more than one composition length can fit inside. Otherwise, it may happen that no train lengths are feasible to choose. In these cases it is not possible to assign a specific composition to train run $j$.

\subsection{Microscopic speed profile model based on dynamic motion equations}

The developed running time model is based on Newton's dynamic motion equations, in which the train is modelled as a mass point. This assumption is widely accepted and used in practice [7], since practical applications have shown satisfactory results. The train length is not neglected in the model since the trajectory of the tail of the train is obtained from the one of the head shifted back for the train length. Referring to the Newton's motion law, the force $f_{s}(v)$ (surplus force) that is used to accelerate a train is produced by the difference between the tractive effort $f_{t}(v)$, and the resistance forces $r(v)$. The tractive effort is generated by the traction unit and applied at the wheel's rim. The resistance forces are obtained as the sum of the resistances due to air viscosity and line characteristics (e.g. gradient and curves). This relation can be formally expressed as:

$$
f_{t}(v)-r(v)=f_{s}(v)=m \cdot d v / d t
$$

The tractive effort is assumed a piecewise function of the train speed $v$ consisting of a linear and a hyperbolic part:

$$
f_{t}(v)=\left\{\begin{array}{lc}
c_{0}+c_{1} v, & v \leq v_{\text {overheat }} \\
c_{2} / v, & v>v_{\text {overheat }}
\end{array},\right.
$$

The linear part of the function $\left(c_{0}+c_{1} v\right)$ is valid for values of the speed lower than the so called overheat speed limit $v_{\text {overheat }}$, while a hyperbolic characteristic is denoted for higher speeds and presents a limitation due to adhesion and tractive power. The resistance 
forces $r(v)$ acting against the train movement are modelled as a second-order polynomial of speed, expressing resistances on a flat and straight line $\left(r_{0}+r_{1} v+r_{2} v^{2}\right)$, and constant resistances due to the topology of tracks, i.e., gradient $\left(f_{G}\right)$ and curve alignment $\left(f_{C}\right)$, respectively:

$$
r(v)=r_{0}+r_{1} v+r_{2} v^{2}+f_{C}+f_{G}
$$

The coefficients $r_{0}, r_{1}$ and $r_{2}$ depend on several variables such as type of the rolling stock, train composition and number and type of train axles. The constant and linear term with coefficients $r_{0}$ and $r_{1}$ represent the mechanical resistance of the rolling stock, while the quadratic term models the aerodynamic resistance. In this model extra resistances relative to the presence of tunnels are not considered. Figure 3 shows a typical trend for the tractive effort and the train resistances as described by (5) and (6), respectively.

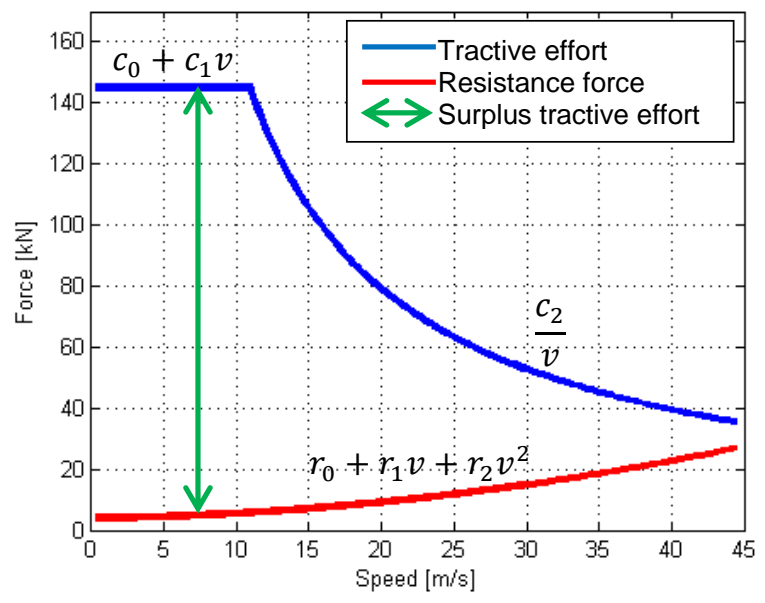

Figure 3. Train characteristics

It should be noted that the mass of the train is implicitly included within the coefficients of resistances and tractive effort equations. Indeed, these are specific coefficients since they are expressed per mass unit. Also, weather conditions such as wind speed, are embodied as part of the parameters. For example, if weather conditions are bad (i.e. wind against train movement direction) this will result in higher resistance parameters.

In order to estimate train trajectories, it is necessary to solve equation (4) for each phase of the motion, that is to say: i) acceleration, ii) cruising and iii) braking. The following characteristics are considered for each phase:

- In the acceleration phase the driver is supposed to accelerate the train by using the tractive effort described by (5) until the train reaches the maximum speed allowed for a given track, or the desired value of cruising speed.

- In the cruising phase the train moves with a constant speed. For a certain track this speed can be the static maximum speed, or a certain lower cruising speed deployed by the train driver. Therefore, the rate between a static speed limit and the cruising speed actually operated is represented by $\theta_{\text {cruising }}$. 
This cruising performance can vary from track to track and depends on the driver behaviour.

- In the braking phase train speed is reduced to accomplish speed restrictions imposed by the track (e.g. static speed limits, switches, stops at stations) or by the signalling system (e.g. red or yellow aspects). Experimental results presented in [12] show that during service two different braking rates are used by trains when 1) slowing down to respect static or dynamic (e.g. given by the signalling system) speed limits and 2) coming to a standstill because of stopping in a station. This assumption has been made in the present model, whereby two braking rates are used for the former $\left(b_{\text {limit }}\right)$ and the latter case $\left(b_{\text {stop }}\right)$, respectively. Specifically, due to the specific allocation of track circuits it has been not possible to collect time data suitable for the determination of $b_{\text {stop }}$. That is why a default value of $0.66 \mathrm{~m} / \mathrm{s}^{2}$ has been used for this parameter as provided by NS.

A partial train trajectory is determined for each phase by computing the speed $v$ assumed by the train at a certain distance $s$, and afterwards calculating the time $t$ corresponding to obtained speed and distance. Particularly, a dynamic train speed profile is modelled as a function of speed depending on distance:

$$
\frac{d v}{d s}=\frac{f_{t}(v)-r(v)}{v}
$$

where $d v / d s$ is the derivative of speed with respect to distance. The corresponding running time is expressed as:

$$
\frac{d t}{d s}=\frac{1}{v}
$$

where $d t / d s$ is the derivative of time to distance. The given equations (7)-(8) are autonomous first-order ordinary differential equations for which several numerical solution methods have been tested in terms of speed and accuracy. As a result, the method given by Dormand-Prince [4] is adopted which is a particular application of the more general Runge-Kutta approach.

\subsection{Formulation of the calibration model: a simulation-based optimization problem} The calibration process is formulated as an optimization problem that aims to minimize the error between simulated and real passage running times. As said actual running times are derived by pre-processing TROTS data.

The decision variables (i.e. the parameters that need to be calibrated) of the problem are listed in Table 1.

Table 1. Decision variables

\begin{tabular}{ll}
\hline$c_{0}$ & maximum starting tractive effort due to overheating limit $[\mathrm{N} / \mathrm{kg}]$ \\
$c_{1}$ & linear parameter of tractive effort equation $[\mathrm{Ns} / \mathrm{m} / \mathrm{kg}]$ \\
$c_{2}$ & hyperbolic parameter of tractive effort function $[\mathrm{Nm} / \mathrm{s} / \mathrm{kg}]$ \\
$r_{0}$ & constant resistance coefficient $[\mathrm{N} / \mathrm{kg}]$ \\
$r_{1}$ & linear resistance coefficient $[\mathrm{Ns} / \mathrm{m} / \mathrm{kg}]$ \\
$r_{2}$ & quadratic resistance coefficient $\left[\mathrm{Ns} / \mathrm{m}^{2} / \mathrm{kg}\right]$ \\
$b_{\text {limit }}$ & braking to speed limit characteristic $\left[\mathrm{m} / \mathrm{s}^{2}\right]$ \\
$\theta_{\text {cruising }}$ & cruising performance [\%] \\
\hline
\end{tabular}


The optimization problem can now be formulated as:

Subject to

$$
\text { Minimize } \sum_{i \in N}\left|t_{i}^{\text {observed }}-t_{i}^{\text {simulated }}\right|
$$

$$
\begin{gathered}
\frac{d v}{d s}=\frac{f_{t}(v)-r(v)}{v} \\
\frac{d t}{d s}=\frac{1}{v} \\
\mathrm{c}_{0} \in\left[\mathrm{c}_{0}^{\mathrm{lb}}, \mathrm{c}_{0}^{\mathrm{ub}}\right] \\
\mathrm{c}_{1} \in\left[\mathrm{c}_{1}^{\mathrm{lb}}, \mathrm{c}_{1}^{\mathrm{ub}}\right] \\
\mathrm{c}_{2} \in\left[\mathrm{c}_{2}^{\mathrm{lb}}, \mathrm{c}_{2}^{\mathrm{ub}}\right] \\
\mathrm{r}_{0} \in\left[\mathrm{r}_{0}^{\mathrm{lb}}, \mathrm{r}_{0}^{\mathrm{ub}}\right] \\
\mathrm{r}_{1} \in\left[\mathrm{r}_{1}^{\mathrm{lb}}, \mathrm{r}_{1}^{\mathrm{ub}}\right] \\
\mathrm{r}_{2} \in\left[\mathrm{r}_{2}^{\mathrm{lb}}, \mathrm{r}_{2}^{\mathrm{ub}}\right] \\
\mathrm{b}_{\text {limit }} \in\left[\mathrm{b}_{\text {limit }}^{\mathrm{lb}}, \mathrm{b}_{\text {limit }}^{\mathrm{ub}}\right] \\
\theta_{\text {cruising }} \in\left[\theta_{\text {cruising }}^{\text {ub }}, \theta_{\text {cruising }}^{\text {ub }}\right] \\
v(0)=v_{0}=0, \quad v(N)=v_{\text {end }}=0 .
\end{gathered}
$$

where the objective function (9) is represented by the absolute error between the simulated and observed passage running times for all the $N$ measurements provided by the TROTS data. It is clear that the evaluation of the objective function requires a preliminary computation of the speed profile and the running time. This means that a numerical integration of the speed and the running time as represented by equations (10) and (11) must be performed at each iteration of the optimization algorithm.

These parameters are respectively relative to the tractive effort equation $\left(c_{0}, c_{1}\right.$ and $\left.c_{2}\right)$, the resistance equation $\left(r_{0}, r_{1}\right.$ and $\left.r_{2}\right)$, the braking rate used to slow down $\left(b_{\text {limit }}\right)$ and the cruising performance adopted by train driver during cruising phases $\left(\theta_{\text {cruising }}\right)$. Equations (12) - (19) define the optimization constraints for each of these variables imposing the lower (lb) and upper bounds (ub) of their domains. Finally, the equation (13) gives the initial and final speed conditions representing that a train starts the run from a standstill and stops at the end of route.

A solution to the optimization problem is therefore represented by the vector:

$$
\beta=\left(c_{0}, c_{1}, c_{2}, r_{0}, r_{1}, r_{2}, b_{\text {limit }}, \theta_{\text {cruising }}\right) \text {, }
$$

which contains a set of values for the decision variables.

\subsection{The optimization metaheuristics: a genetic algorithm}

A genetic algorithm (GA) is developed to solve the optimization problem. GA is a wellknown robust and adaptive method largely used in the scientific field to solve search and optimization problems. The algorithm works with a population of individuals, each representing a possible solution, in this case a set of train parameters $\beta$. Each individual 
produces a different value of the objective function. The population evolves towards better solutions (i.e. lower values of the objective function) by means of randomized processes of selection, crossover, and mutation (see [13] for more information on the topic). The GA used in this paper has been developed in Matlab and customized to improve its performances according to the specific problem applied. Moreover, its execution has been parallelized by allocating different functions of the algorithm to different threads. This strongly reduces computing times of the optimization when adopting multi-core computers.

\section{Case study: the Rotterdam-Delft corridor}

The framework proposed in this research has been applied to calibrate a significant set of trains running along the corridor Rotterdam-Delft, which is one of the most densely operated lines in the Netherlands. The line has a length of $14.3 \mathrm{~km}$ with a double track layout. The Dutch speed signalling system NS' 54 with ATB automatic train protection is implemented over the whole corridor. A detailed explanation of this system can be found in [1]. Both regional and Intercity (IC) trains operate on this line, but for the sake of simplicity the analysis performed in this research is only demonstrated to the latter type of trains.

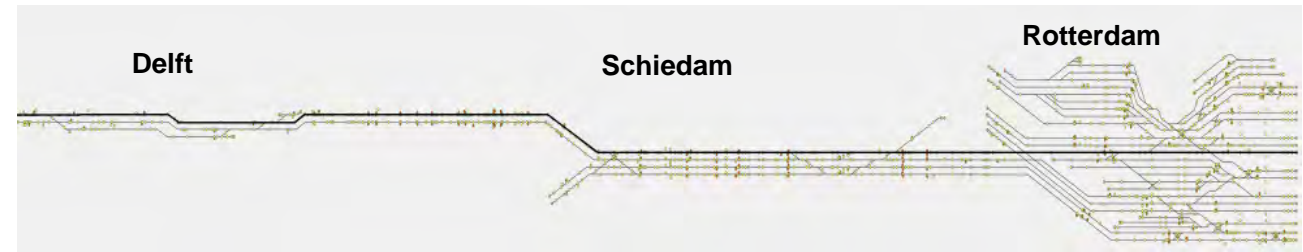

Figure 4. Schematic layout of the corridor Rotterdam - Delft

In particular, the intercity train line IC1900 is analysed. According to the planned timetable, the rolling stock used in service is reported in Table 2. Four different classes of train compositions have been observed: the electrical multiple units VIRM with four and six and ten units as well as the locomotive hauled trains ICRm with ten cars. All these trains use the same route and therefore the same platform tracks, in- and outbound interlocked routes, and block sections, with a slight difference in terms of the stop locations in stations.

Table 2: Input data of rolling stock

\begin{tabular}{lcc}
\hline Train composition & Length $[\mathrm{m}]$ & $\mathrm{v}_{\max }[\mathrm{km} / \mathrm{h}]$ \\
\hline VIRM4 & 108 & 160 \\
VIRM6 & 162 & 160 \\
VIRM10 & 270 & 160 \\
ICRm (Locomotive $1700+10$ cars) & 282 & 160 \\
\hline
\end{tabular}

The calibration of the running time model is performed for observed TROTS data collected over a seven-day period of operations in April 2010. In total 42 track sections have been considered. This means that the parameters of each train run have been calibrated versus 42 time-distance observations.

All the calibration experiments are carried out on an AMD Athlon $3300 \mathrm{GHz}$ 
processor with six cores and 4GB of RAM. The integration of a single train trajectory takes about 0.02 seconds, while the computing time needed to complete a single calibration experiment is always lower than one minute.

\subsection{Analysis of parameters and model performance}

A preliminary sensitivity analysis has been performed to understand which input parameters is the more influential for the running time model. This has been carried out by evaluating variation of the running time model output by changing the value of one parameter while keeping fixed the other ones. Such procedure has been repeated for all input elements. Only the parameters that produced a significant variation of the running time has been selected for the calibration, since the model is more sensitive to these ones. In particular, the linear parameter of the resistance equation, $r_{1}$, does not have significant importance, given that this parameter produces a variation of running times less than $0.1 \%$. Lukaszevicz [11] came to the same conclusion for passenger trains. A scarce relevance is also identified for the linear parameter of the tractive force equation, $c_{1}$. Fixed values have been assumed for these two parameters, therefore the calibration process has been reduced to the following factors:

$$
\beta=\left(c_{0}, c_{2}, r_{0}, r_{2}, b_{\text {limit }}, v_{\text {cruising }}\right) \text {. }
$$

As a result, the value of $c_{1}$ has been set to zero, while $r_{1}$ is fixed to the default value used by the RU (given by the rolling stock input data) and dependent on the train length.

A stability analysis has been carried out to evaluate the robustness and the performances of the optimization algorithm. In particular, 30 calibration experiments have been executed for a fixed realised train trajectory. This allows to understand if the algorithm is able to return the consistent results for the same calibration problem (with the same observed data). If the value returned for each parameter is not the same over the different experiments then the algorithm is not robust enough and/or the optimization problem is not well-defined. Results obtained from this test are reported in Table 3 which shows the average and the standard deviation of the values determined for each parameter over the 30 calibration experiments. It can be seen that parameters $c_{2}, b_{\text {limit }}$ and $\theta_{\text {cruising }}$ converge to the same value for all the calibration experiments. Relatively low values of the standard deviation are observed for $r_{0}$ and $r_{2}, 4.24 \%$ and $2.79 \%$, respectively. However, variations of these two parameters produce just slight changes in the objective function value and the total running time of $0.4 \%$ and $0.03 \%$, respectively. This outcome confirms the robustness of the algorithm used and the validity of the formulated optimization problem.

Table 3. Model performance output

\begin{tabular}{lcccc}
\hline \multirow{2}{*}{ Parameter } & $\begin{array}{c}\text { Given } \\
\text { value }\end{array}$ & $\begin{array}{c}\text { Average } \\
\text { value }\end{array}$ & $\begin{array}{c}\text { Standard Deviation } \\
\text { Value }\end{array}$ & $\%$ \\
\hline$c_{0}\left[10^{-4} \mathrm{~N} / \mathrm{kg}\right]$ & 5.62 & 5.33 & 0.05 & 0.96 \\
$c_{2}[\mathrm{Nm} / \mathrm{s} / \mathrm{kg}]$ & 6.21 & 6.29 & 0.00 & 0.00 \\
$r_{0}\left[10^{-2} \mathrm{~N} / \mathrm{kg}\right]$ & 1.53 & 1.60 & 0.07 & 4.24 \\
$r_{2}\left[10^{-5} \mathrm{Ns} / \mathrm{m}^{2} / \mathrm{kg}\right]$ & 4.08 & 3.55 & 0.10 & 2.79 \\
$b_{\text {limit }}\left[\mathrm{m} / \mathrm{s}^{2}\right]$ & 0.66 & 0.24 & 0.00 & 0.00 \\
$\theta_{\text {cruising }}[\%]$ & 100 & 101 & 0.00 & 0.00 \\
Objective function $[\mathrm{s}]$ & 135.76 & 79.00 & 0.34 & 0.42 \\
Running time $[\mathrm{s}]$ & 595.8 & 572.64 & 0.18 & 0.03 \\
\hline
\end{tabular}


Consecutively, the parameters are compared with the corresponding default values provided by the RU (second column of Table 3 ). As can be seen the calibrated values vary around the default ones for all the parameters but the braking rate $b_{\text {limit }}$. The latter is due to the fact that during the observed train run a the train driver adopted a braking rate that was on average lower than the one assumed by the operating company. Therefore, such aspect highlights the ability of the proposed model to estimate also the driving behaviour of the train driver.

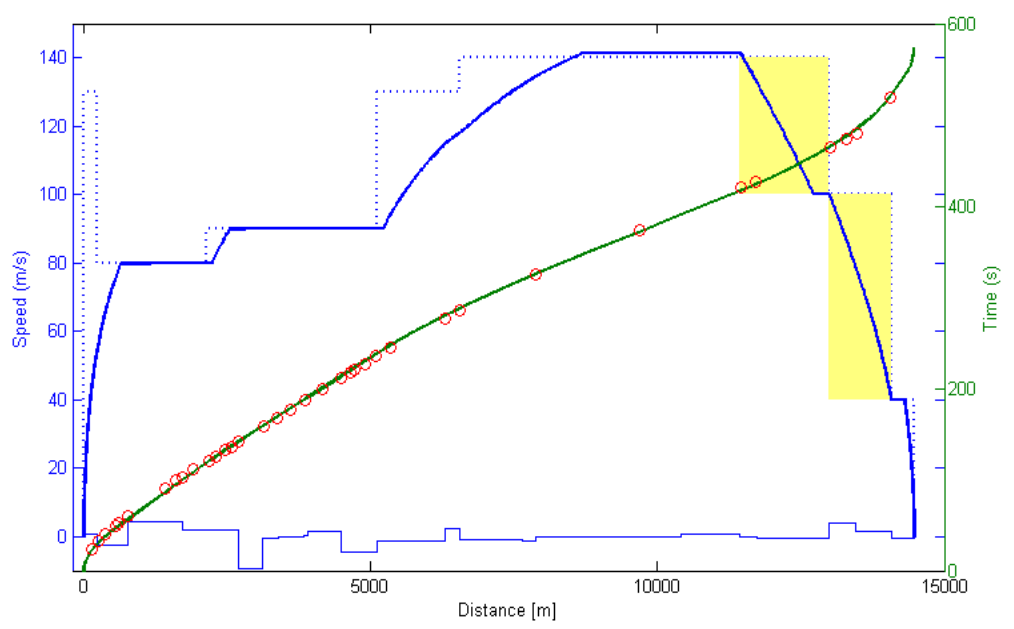

Figure 5. Estimated speed profile and time-distance diagram for a single train run

Figure 5 illustrates the outputs of a single calibration experiment: the calibrated distance-speed diagram (solid blue line) and the corresponding time-distance trajectory (solid green line). Red circles depict measured time-distance as given by TROTS. The effectiveness of the calibration performed is immediately visible since the simulated timedistance trajectory practically overlaps observed data. This means a very high accuracy of the model. The gradient profile of the track is reported with the blue line at the bottom while the static speed limit is depicted with the dashed blue line. Yellow blocks represent the approach indication corresponding to those block sections in which trains has to start braking because of a restricted aspect imposed by the NS'54/ATB system.

\subsection{Train length estimation}

The train lengths are estimated by means of the process explained in section 3.3. Figure 4 shows the obtained intervals for the train lengths of the observed trains. Horizontal lines show the width of this interval for each train run, while vertical lines indicate the four lengths associated to each of the four compositions considered. A different colour has been used to represent the estimated length. Magenta is adopted for the class VIRM4, green for VIRM6, blue for VIRM10, red for ICRm, while grey is employed to represent cases in which it was not possible to have a correct estimation of train length (i.e. when no composition length falls inside the interval). 

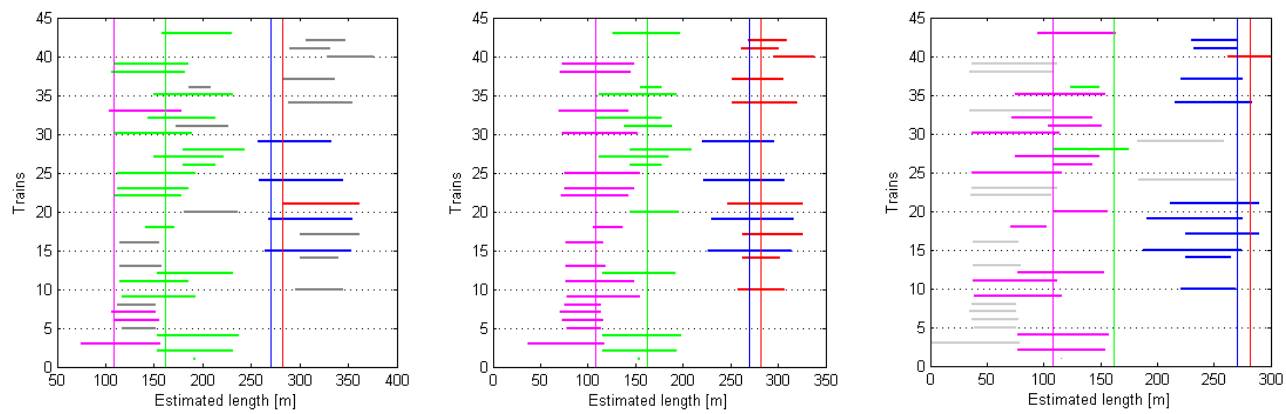

Figure 6. Estimation of trains lengths for: a) measured release times, b) measured release times with delay of one second and c) measured release times with delay of two seconds

As can be seen these train length intervals have different ranges. This depends on the value of the error $\delta$ with which the occupation and release times are measured and the value estimated for the average train speed $v$ adopted on the track section. Hence, based on the data of realised rolling stock, it was possible to assert part of the estimated train lengths, and therefore to distinguish the most probable $\delta$ which is 1 second in this case (Figure 6b).

\subsection{Calibration Results}

Calibration of parameters is undertaken for the four classes of train compositions. For each class 16 train runs have been examined. Parameters of the running time model have been calibrated for each of the train runs. This means that 16 sets of calibrated parameters $\beta$ is provided for each train composition. This consents to estimate variations of a certain parameter over different train runs for a given composition. In this way a probability distribution has been assessed for each parameter by applying the method of the maximum likelihood estimation (MLE). Figures 7-9 shows the results obtained for a train class VIRM4. In particular, for each input factor it is reported the corresponding probability distribution and the related distribution parameters. It should be noted that similar distributions are obtained for the other compositions and for brevity they are not explicitly reported.

Figure $7 \mathrm{a}$ gives the distribution of the constant parameter of the tractive effort. It shows that this parameter fits best to a generalized extreme value (GEV) distribution. As expected, not all the observed train runs use the maximum tractive effort while accelerating from a standstill. Nevertheless, some runs exceeded the theoretical maximum tractive force given by the RU. Figure $7 \mathrm{~b}$ shows that parameter $c_{2}$ fits best to the Gumbel minimum distribution. It is observed that in a certain number of train runs $c_{2}$ was higher than the experimental maximum, which is clearly expressed by the mode value of the fitted distribution. 


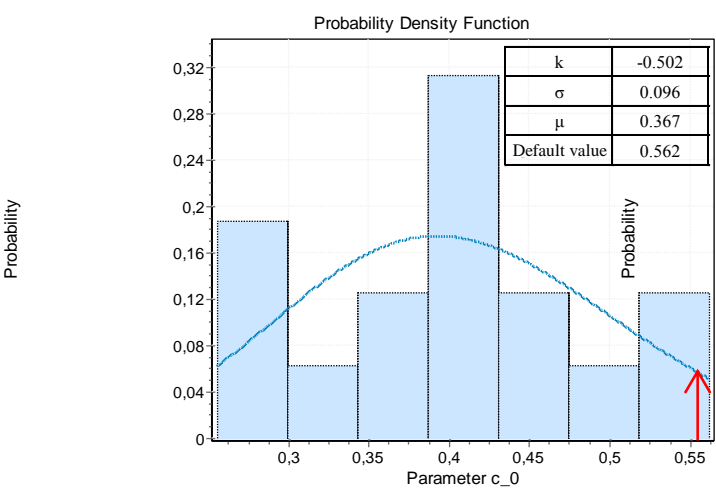

$\square$ Histogram - Gen. Extreme Value

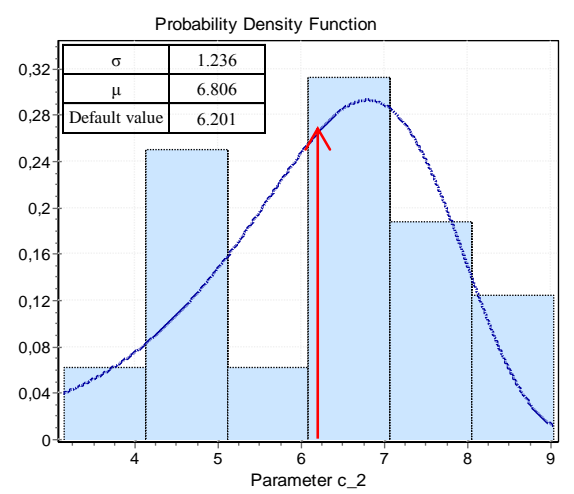

$\square$ Histogram - Gumbel Min

Figure 7. Distributions of tractive effort parameters

The constant parameter of the resistance equation $r_{0}$ (Figure 8a) shows a good fit to a log-logistic distribution. It can be observed that calibrated estimates tend to undervalue the theoretical value. The distribution of $r_{0}$ can be explained by recent developments in rail-wheel contact and consequently, expected reduction of mechanical resistance. Nevertheless, the mean value equals 0.0155 and therefore shows good correspondence to the default value. On the other hand, higher values may be an effect of deteriorated rolling stock. The quadratic parameter $r_{2}$ shows the best fitting to a Frechet distribution. From Figure $8 \mathrm{~b}$ it can be distinguished the variance of the aerodynamic resistance, which may be considerable while taking into account adverse weather conditions. Thereby, it may be assumed that the default value is slightly overestimated.
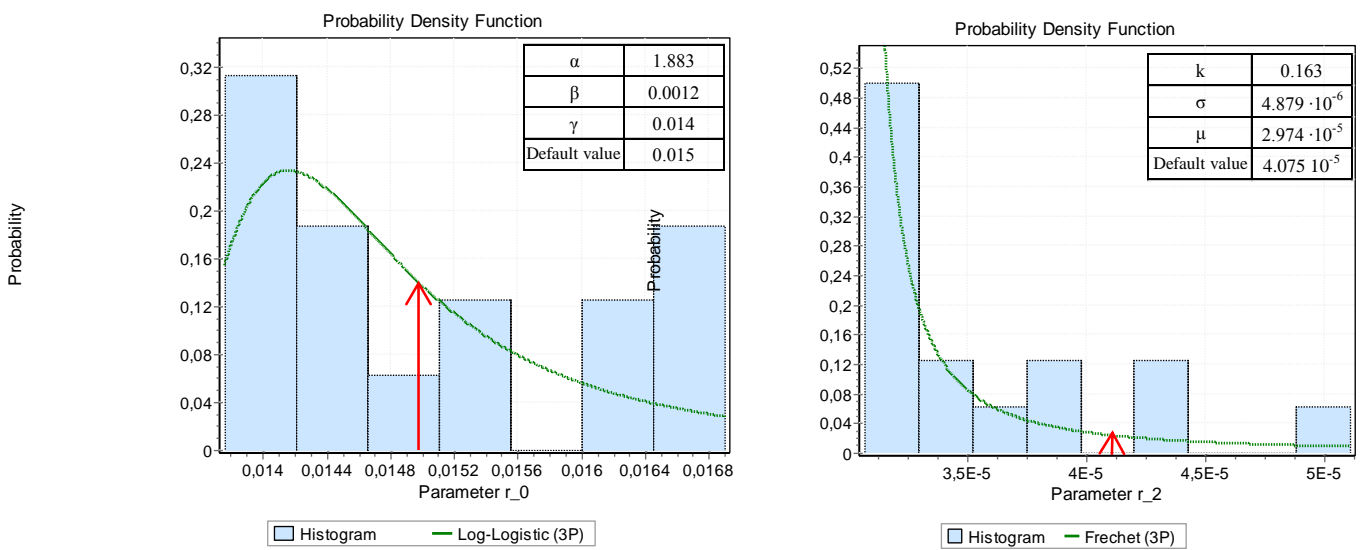

Figure 8. Distributions of resistance parameters

The distribution of the braking rate (due to speed restriction) is shown in Figure 9a. It can be observed that the most probable rate is significantly less than the default value used by the RU which is $0.66 \mathrm{~m} / \mathrm{s}^{2}$. Some of the higher values of the parameter can be evaluated as an error in calibrated parameters regarding the inability of the current model to detect and reconstruct coasting phases. For example, in case of coasting, a simulated train speed profile tends to have a higher speed at the approach indication than the realised 
train behaviour with coasting. The braking parameter shows the best goodness of fit with the distribution of Frechet, which belongs to a family of the GEV distributions. This parameter shows a relevant variation over the different train runs. An explanation to this can be the consistent difference in the driving behaviour for different train drivers.

Finally, the cruising performance is depicted in figure $9 \mathrm{~b}$. It clearly shows that trains tend to run on the maximum allowable speed given the by static speed limit while some of them even overrun this limit for 1-2\%. This parameter fits best to GEV distribution.
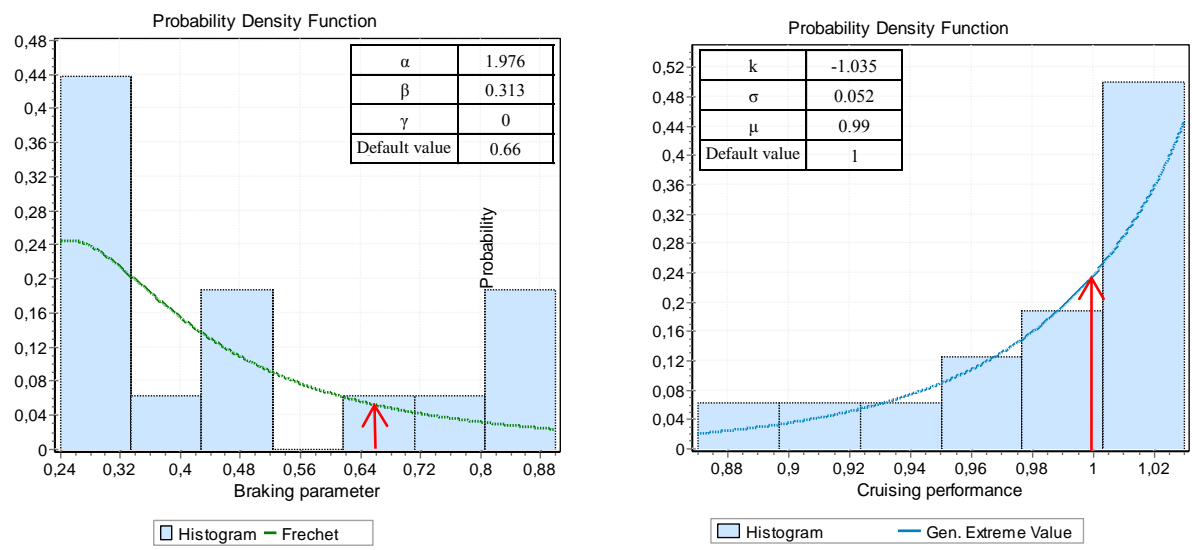

Figure 9. Parameter distributions for: a) braking rate, b) cruising performance

Table 4 present the ranges of calibrated parameters for all the train compositions. Parameter $r_{1}$ is not given as an interval since it was not part of the calibration and set to a fixed default value as provided by the RU.

Table 4. Calibrated parameters for the four train compositions

\begin{tabular}{lcccc}
\hline & VIRM4 & VIRM6 & VIRM10 & ICRm \\
\hline$c_{0}\left[10^{-3} \mathrm{~N} / \mathrm{kg}\right]$ & {$[0.251,0.621]$} & {$[0.440,0.600]$} & {$[0.200,0.511]$} & {$[0.283,0.503]$} \\
$c_{2}[\mathrm{Nm} / \mathrm{s} / \mathrm{kg}]$ & {$[3.144,8.648]$} & {$[3.669,7.075]$} & {$[2.555,7.355]$} & {$[3.341,11.792]$} \\
$r_{0}[\mathrm{~N} / \mathrm{kg}]$ & {$[0.014,0.016]$} & {$[0.014,0.016]$} & {$[0.010,0.020]$} & {$[0.019,0.022]$} \\
$r_{1}\left[10^{-4} \mathrm{Ns} / \mathrm{m} / \mathrm{kg}\right]$ & 2.162 & 1.939 & 3.341 & 3.342 \\
$r_{2}\left[10^{-5} \mathrm{Ns}^{2} / \mathrm{m}^{2} / \mathrm{kg}\right]$ & {$[3.499,4.678]$} & {$[2.910,3.904]$} & {$[1.774,3.597]$} & {$[2.672,3.616]$} \\
$b_{\text {limit }}\left[\mathrm{m} / \mathrm{s}^{2}\right]$ & {$[0.24,0.9]$} & {$[0.24,0.9]$} & {$[0.24,0.9]$} & {$[0.24,0.9]$} \\
$v_{\text {cruising }}[\mathrm{m} / \mathrm{s}]$ & {$[0.89,1.02]$} & {$[0.81,1.02]$} & {$[0.89,0.98]$} & {$[0.81,1.02]$} \\
\hline
\end{tabular}

Figure 10 illustrates results from Table 4 and gives a comparison with the default values of parameters provided by the RU. As can be observed the default values (yellow dots) represent neither the upper bound nor the average value of the distributions of the calibrated input parameters. This aspect can be clearly seen for the factors relative to the tractive effort, $c_{0}$ and $c_{2}$. The default values given by the RU for these parameters are usually employed for the calculation of the minimum running time, and therefore should represent the upper bound of these intervals since it is assumed that the train accelerates with the maximum power of the engine. Instead, the results of the calibration experiment show the presence of train runs that overcome these values in the reality. Furthermore, parameters of the resistance equation, $r_{0}$ and $r_{2}$, supplied by the RU are within the estimated distributions, for all the train compositions. For $r_{0}$ was expected to be the lower 
bound of these intervals. Parameter $r_{2}$ describes the aerodynamic resistances and takes into account the effect of the wind in the same or the opposite direction of the train run. The expectations were that the default values supplied for these parameters tallied with the means of the corresponding distributions. Nevertheless, it has been observed that the default values tend to represent a slightly overestimated values comparing with the observed distributions. On the other hand, the large variation intervals are revealed for the braking rate. This denotes a consistent variation in the driving behaviour of train drivers. The default value for braking rate cannot describe this aspect. Moreover, this value does not coincide with any representative value of the distribution (i.e. mean, lower or upper bound). For the cruising performance the same conclusions can be drawn as the braking rate.

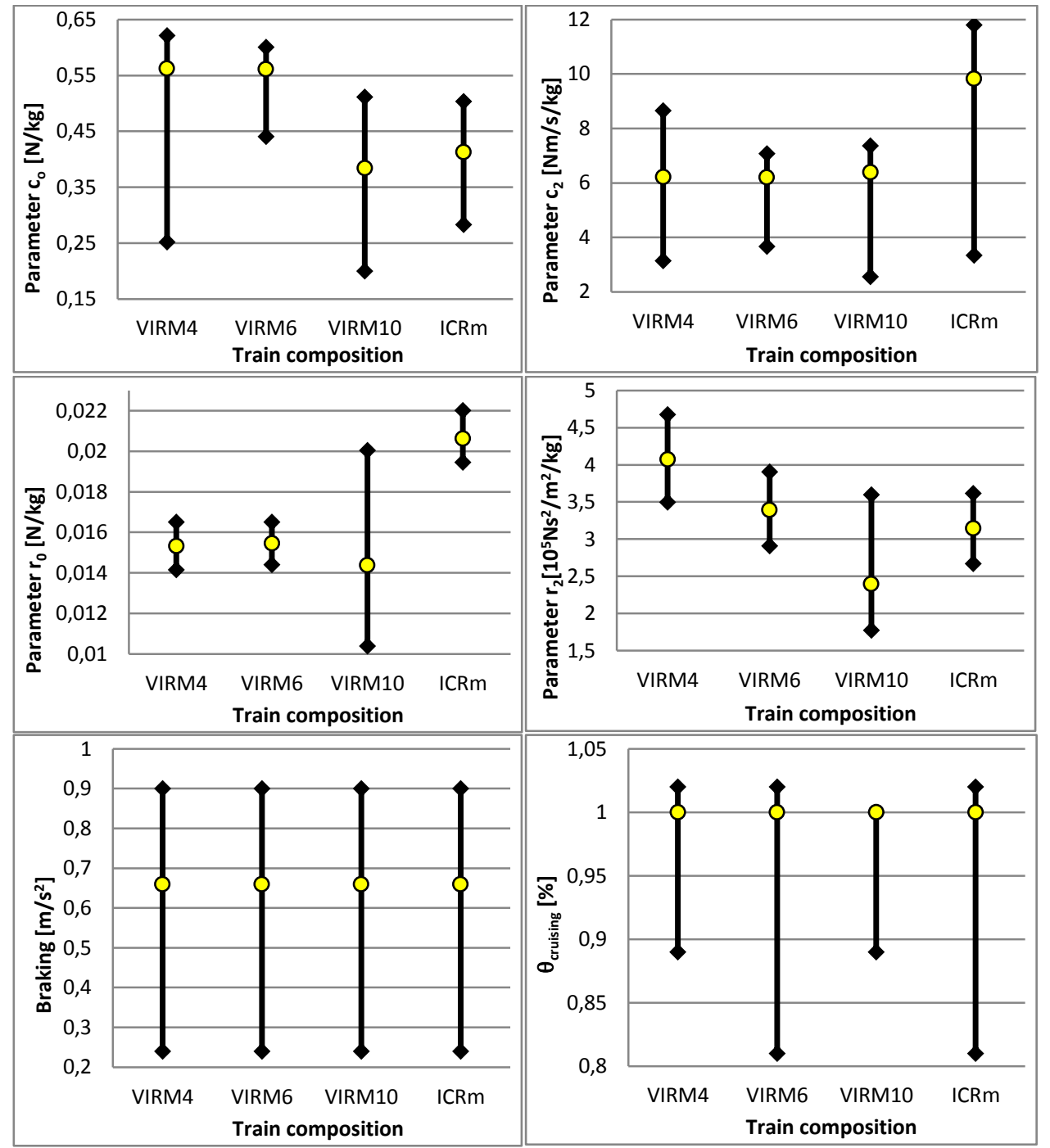

Figure 10. Calibrated parameters for the four train composition 


\section{Conclusions}

Predictions of railway traffic are needed by designers and dispatchers respectively for the design of robust timetables and the real-time management of perturbed conditions. These tasks can be performed effectively only when using train running time models which reliably describe actual trajectories. To this purpose the calibration of model parameters against field data is necessary.

This paper presents an approach to derive the most probable speed profiles of train runs from observed track occupation/release data. The train behaviour is modelled according to the Newton dynamic motion equations, which are numerically integrated over distance employing the Runge-Kutta method. A simulation-based optimization approach is adopted to calibrate input parameters of the equations describing the tractive effort, the motion resistances, the braking effort, and the cruising phase. These parameters are fine-tuned for different classes of train composition (defined by the number of wagons, the type of traction unit, and the length of the train) by minimizing the error between observed and simulated running times, using a genetic algorithm. For each composition the calibration experiment is performed on a significant set of observed trains running along the Rotterdam-Delft corridor in the Netherlands. A probability distribution has been estimated for the input parameters of each class of composition. This aspect gives also insights in different driving behaviour adopted during real operations.

The results show that the train length estimation model obtained good computation accuracy. To this aim the error due to the delay of the release time has been distinguished. Further, the results illustrate the effectiveness of the proposed optimization method in calibrating parameters of the Newton's dynamic equations versus track occupation/release data collected at the level of track sections. It has been observed that some of the parameters of tractive effort and resistance do not affect the train behaviour significantly, i.e., the linear parameter of tractive effort as well as the linear parameter of resistance force. Furthermore, the comparison with the default parameters provided by the RU highlights that some of the default values tend to be inadequate for the calculation of the technical running time for which they are generally used. Tractive effort parameters seldom overreach the corresponding default values, therefore showing that the latter are not the absolute maximum values, but a train has an extra power reserve that can be used for faster running. On the other hand, the parameters of the resistance equation tend to be slightly overestimated based on the received distributions. The realised braking rate is significantly smoother than the default one; therefore trains traverse the braking distance faster than computed in the minimum running time. Also, train drivers do not always follow the maximum static speed limit. Instead, it has been observed that in some cases the cruising performance is just $80 \%$ of the maximum. Finally, it has been shown that a specific calibration process should be performed to understand the variation in the coefficients of the dynamic motion equations over different train runs. In this way it is possible to set more reliable values to generate stochastic running times during robust timetabling.

The current work can be extended in several ways. First, the calibration model could be performed on different lines to evaluate possible different behaviour of train drivers as well as to distinguish parameters for different train compositions. Second, it would be noteworthy to compare realised and simulated running times based on achieved stochastic parameters and analyse the differences between them. Third, the computation time of the proposed simulation-based model can be enhanced by adjusting parameters of the implemented GA. Moreover, an improvement of the speed profile simulation model may 
be carried out in order to represent the driver behaviour more accurately. A proper validation of the speed profiles obtained by this model will be realized against GPS data.

\section{References}

[1] Albrecht T., Gassel C., Binder A., van Luipen J. (2010). Dealing with operational constraints in energy efficient driving. Proceedings of the 4th IET International Conference on Railway Traction Systems (RTS 2010), Birmingham.

[2] Albrecht T., Gassel C., Knijff J., van Luipen, J. (2010). Analysis of energy consumption and traffic flow by means of track occupation data. Proceedings of the 4th IET International Conference on Railway Traction Systems (RTS 2010), Birmingham.

[3] Albrecht T., Goverde R.M.P., Weeda V.A., Van Luipen J. (2006). Reconstruction of train trajectories from track occupation data to determine the effects of a Driver Information System. In: Allan J., Brebbia C.A., Rumsey A.F., Sciutto G., Sone S., (eds.), Computers in Railways X, WIT Transactions on The Built Environment, 88, WIT Press, Southampton, pp. 207-216.

[4] Butcher J.C. (2003). Numerical Methods for Ordinary Differential Equations. Wiley, London.

[5] Daamen W., Goverde R.M.P., Hansen I.A. (2009). Non-Discriminatory Automatic Registration of Knock-On Train Delays. Networks and Spatial Economics, 9(1), 4761.

[6] Goverde R.M.P., Meng L. (2011). Advanced monitoring and management information of railway operations. Journal of Rail Transport Planning and Management, 1(2), 69-79.

[7] Hansen I.A., Pachl J. (2008). Railway Timetable and Traffic. Eurailpress, Hamburg.

[8] Hertel G., Steckel J. (1992). Fahrzeitberechnung unter stochastischem Aspect. Eisenbahningenieur, 43(5), 304-306.

[9] Kecman P., Goverde R.M.P. (2012). Process mining of train describer event data and automatic conflict identification. In: Brebbia C.A., Tomii N., Tzieropoulos, P., Mera J.M., (eds.), Computers in Railways XIII, WIT Transactions on The Built Environment, 127, WIT Press, Southampton, pp. 227-238.

[10] Kecman P., Goverde R.M.P. (2013). Process Mining Approach for Recovery of Realized Train Paths and Route Conflict Identification. The 92st Annual Meeting of Transportation Research Board, Washington, D.C.

[11] Lukaszevicz P. (2001). Energy Consumption and Running Time for Trains. PhD Thesis, Royal Institute of Technology, Stockholm.

[12] Medeossi G., Longo G., de Fabris S. (2011). A method for using stochastic blocking times to improve timetable planning. Journal of Rail Transport Planning \& Management, 1(1), 1-13.

[13] Mitchell M. (1996). An Introduction to Genetic Algorithms. MIT Press, Cambridge, MA.

[14] ProRail (2008). TROTS protocol - interface design description (in Dutch). Utrecht, 2008. 
[15] Radosavljevic A. (2006). Measurement of train traction characteristics. Proceedings of the Institution of Mechanical Engineers, Part F: Journal of Rail and Rapid Transit, 220(3), 283-291. 